\title{
Geometric Mean Cordial Labeling of Transformation Graph of a Star Graph
}

\author{
K. Nagarajan, K. Chitra Lakshmi
}

Abstract: Let $G=(V, E)$ be a graph and f be a mapping from $V(G) \rightarrow\{0,1,2\}$. For each edge uv, assign the label $|\sqrt{f(u) f(v)}|, f$ is called a geometric mean cordial labeling if $\left|v_{f}(i)-v_{f}(j)\right| \leq 1$ and $\left|e_{f}(i)-e_{f}(j)\right| \leq 1$, where $v_{f}(x)$ and $e_{f}(x)$ denote the number of vertices and edges labeled with $x, x \in$ $\{0,1,2\}$ respectively.

A graph with a geometric mean cordial labeling is called geometric mean cordial graph. In this paper, the geometric mean cordiality of transformation graph of star is discussed.

Keywords: Cordial labeling, cordial graph, geometric mean cordial labeling, geometric mean cordial graph, transformation graph.

\section{INTRODUCTION}

We use the symbol $\Gamma x\rceil$ denotes the smallest integer greater than or equal to x. Some terms are used in the sense of Harary, Bondy and Murthy [2, 5]. The concept of cordial labeling [3] was introduced by Cahit in the year 1987. The labeled graphs are applied mostly in the areas of radar, circuit design, communication network, astronomy, cryptography etc [4].

For a graph $G=(V, E)$, let $f: V(G) \rightarrow\{0,1\}$ be a function. For each edge $u v$, assign the label $|f(u)-f(v)|, f$ is called a cordial labeling if $\left|v_{f}(i)-v_{f}(j)\right| \leq 1$ and $\left|e_{f}(i)-e_{f}(j)\right| \leq 1$, where $v_{f}(x)$ and $e_{f}(x)$ denote the number of vertices and edges labeled with $x, x \in\{0,1\}$ respectively.

A graph which admits cordial labeling is called is a cordial graph. Mean cordial labeling was introduced by R. Ponraj, M. Sivakumar and M. Sundaram [6].

We defined geometric mean cordial labeling in [ 7 ]. In the previous six papers $[7,8,9,10,11,12]$, we have checked the geometric mean cordiality of standard graphs, subdivision of standard graphs, corona of standard graphs, transformation graphs of paths and cycles.

In this paper, the geometric mean cordiality is checked for the transformation graph of a star.

Revised Manuscript Received on December 15, 2019.

Dr. K. Nagarajan, Head and Associate Professor, Department of mathematics at Sri S. Ramasamy Naidu Memorial College, Sattur, India.

K. Chitra Lakshmi, Assistant Professor, Department of Mathematics, V. V. Vanniaperumal College for Women, Virudhunagar.

\section{PRELIMINARIES}

First we define a transformation graph of a graph.

Definition 2.1.[11] A transformation graph $G^{x y z}$ is the graph whose vertex set is $V(G) \cup E(G)$. Two of its vertices are adjacent if and only if their associativity is consistent with the corresponding elements of $x y z$. Let $\alpha$ and $\beta$ be two elements of $V(G) \cup E(G)$. Then the associativity of $\alpha$ and $\beta$ is taken as + if they are adjacent or incident in $G$, otherwise -. Let $x y z$ be the 3 - permutation of the set $\{+,-\}$. The pair $\alpha$ and $\beta$ is said to correspond to $x$ or $y$ or $z$ if both $\alpha$ and $\beta$ are in $V(G)$ or both in $E(G)$ or one is in $V(G)$ and the other is in $E(G)$ respectively.

Theorem 1.2: $P_{n}^{+++}$is geometric mean cordial if and only if $n \equiv 1(\bmod 3)$.

Theorem 1.3: $P_{n}^{+{ }^{+}}$is geometric mean cordial if and only if $n \equiv 1(\bmod 3)$.

Theorem 1.4: $P_{n}{ }^{++}$is geometric mean cordial if and only if $n \equiv 1,2(\bmod 3)$.

Theorem 1.5: $P_{n}^{-{ }^{-+}}$is geometric mean cordial.

Theorem 1.6: $C_{n}{ }^{++}$is not geometric mean cordial.

Theorem 1.7: $C_{n}^{+{ }^{+}}$is not geometric mean cordial.

Theorem 1.8: $C_{n}{ }^{-+}$is geometric mean cordial.

Theorem 1.9: $C_{n}^{-{ }^{+}}$is not geometric mean cordial.

\section{MAIN RESULT}

Here, we will prove transformation graph of a star is not geometric mean cordial.

Theorem 3.1. $K_{1, n}{ }^{++}$is not geometric mean cordial.

Proof: Let $K_{1, n}$ be the star graph of $n+1$ vertices and $n$ edges. Then $K_{l},{ }_{n}{ }^{+}+$is a graph of $2 n+1$ vertices and $\frac{5 n+n^{2}}{2}$ edges.

This graph can be drawn so that it is decomposed into 3 edge disjoint graphs $G_{l}, G_{2}$ and $G_{3}$ such that $G_{l}$ and $G_{2}$ are isomorphic to $K_{l, n}$ and $G_{3}$ is isomorphic to $K_{n} \odot K_{l}$.

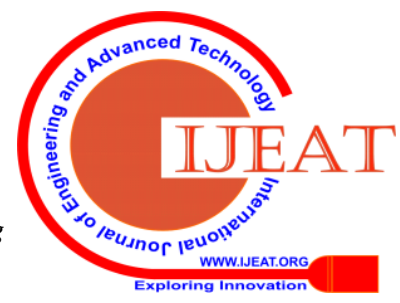




\section{Geometric Mean Cordial Labeling of Transformation Graph of a Star Graph}

Case $(\mathbf{i}): n \equiv 0(\bmod 3)$. Let $n=3 t$.

Now, the graph $K_{1, n}+++$ has $6 t+1$ vertices and $\frac{15 t+9 t^{2}}{2}$ edges. We have 3 subcases.

(i) $v_{f}(0)=v_{f}(2)=2 t, \quad v_{f}(1)=2 t+1$

(ii) $v_{f}(0)=v_{f}(1)=2 t, v_{f}(2)=2 t+1$.

(iii) $v_{f}(1)=v_{f}(2)=2 t, v_{f}(0)=2 t+1$ and

$e_{f}(0)=e_{f}(1)=e_{f}(2)=\frac{5 t+3 t^{2}}{2}$. $-(1)$

Subcase $(\mathbf{i}): v_{f}(0)=v_{f}(2)=2 t, v_{f}(1)=2 t+1$.

Now, we consider the subgraphs $G_{1}, G_{2}$ and $G_{3}$ of $K_{l, n}+++$. We label the central vertex as 1 , label all the other vertices of $G_{1}$ and $G_{2}$ of $K_{l, n}+++$ as in the case (i) of Theorem 2.7[7],. It follows that, the graphs $G_{l}$ and $G_{2}$ together gives the edge contributions as

$$
e_{f_{G_{1} \cup G_{2}}}(0)=e_{f_{G_{1} \cup G_{2}}}(1)=e_{f_{G_{1} \cup G_{2}}}(2)=2 t
$$

and also from case (i) of Theorem,2.1 [11], it follows that the graph $G_{3}$ gives the edge contributions as

$e_{f_{G_{3}}}(0)=\left(\begin{array}{l}t \\ 2\end{array}\right)+t^{2}+t^{2}+t, e_{f_{G_{3}}}(1)=\left(\begin{array}{l}t \\ 2\end{array}\right)+t$,

$e_{f_{G_{3}}}(2)=\left(\begin{array}{l}t \\ 2\end{array}\right)+t^{2}+t$. Totally, we have

$e_{f}(0)=\frac{5 t+5 t^{2}}{2} e_{f}(1)=\frac{5 t+t^{2}}{2} e_{f}(2)=\frac{5 t+3 t^{2}}{2}$.

In this subcase, if we reduce the contribution $t^{2}$ from $e_{f}(0)$ and add the contribution $t^{2}$ to $e_{f}(1)$, we get the condition (1). But, this removal affects the vertex labeling condition (i).

Subcase $($ ii $): v_{f}(0)=v_{f}(1)=2 t, v_{f}(2)=2 t+1$.

Suppose the central vertex is labeled as 2 . We apply the similar argument in subcase (i) of case (i), we get $e_{f_{G_{1} \cup G_{2}}}(0)=2 t, e_{f_{G_{1} \cup G_{2}}}(1)=0, e_{f_{G_{1} \cup G_{2}}}(2)=4 t$,

$e_{f_{G_{3}}}(0)=\left(\begin{array}{l}t \\ 2\end{array}\right)+t^{2}+t^{2}+t, e_{f_{G_{3}}}(1)=\left(\begin{array}{l}t \\ 2\end{array}\right)+t$

$e_{f_{G_{3}}}(2)=\left(\begin{array}{l}t \\ 2\end{array}\right)+t^{2}+t$. Totally, we have

$e_{f}(0)=\frac{5 t+5 t^{2}}{2} e_{f}(1)=\frac{t+t^{2}}{2} e_{f}(2)=\frac{9 t+3 t^{2}}{2}$.

In this subcase, if we reduce the contribution $t^{2}$ from $e_{f}(0)$ and $2 t$ from $e_{f}(2)$ add the contribution $t^{2}+2 t$ to $e_{f}$ (1), we get the condition (1). But, this removal affects the vertex labelingcondition (ii).

Subcase ( iii ): $v_{f}(1)=v_{f}(2)=2 t, \quad v_{f}(0)=2 t+1$.

Suppose the central vertex is labeled 0 . We apply the similar argument in subcase (ii) of case (i), we get

$$
\begin{aligned}
& e_{f_{G_{1} \cup G_{2}}}(0)=6 t, e_{f_{G_{1} \cup G_{2}}}(1)=0, e_{f_{G_{1} \cup G_{2}}}(2)=0, \\
& e_{f_{G_{3}}}(0)=\left(\begin{array}{l}
t \\
2
\end{array}\right)+t^{2}+t^{2}+t, e_{f_{G_{3}}}(1)=\left(\begin{array}{l}
t \\
2
\end{array}\right)+t,
\end{aligned}
$$

$e_{f_{G_{3}}}(2)=\left(\begin{array}{l}t \\ 2\end{array}\right)+t^{2}+t$. Totally, we have

$e_{f}(0)=\frac{13 t+5 t^{2}}{2} e_{f}(1)=\frac{t+t^{2}}{2} e_{f}(2)=\frac{t+3 t^{2}}{2}$.

In this subcase, if we reduce the contribution $t^{2}+4 t$ from $e_{f}(0)$ add the contribution $t^{2}+2 t$ to $e_{f}(1)$ and $2 t$ to $e_{f}$ (2), we get the condition (1). But, this removal affects the vertex labeling condition (iii).

Case (ii): $n \equiv 1(\bmod 3)$. Let $n=3 t+1$.

Now the graph $K_{1, n}+++$ has $6 t+3$ vertices and $\frac{6+21 t+9 t^{2}}{2}$ edges. We have only one subcase.

$v_{f}(0)=v_{f}(1)=v_{f}(2)=2 t+1$

$e_{f}(0)=e_{f}(1)=e_{f}(2)=\frac{2+7 t+3 t^{2}}{2}$

From subcase (iii) of case (ii) of Theorem 2.1 [11]

it follows that the graph $G_{3}$ gives the edge contributions as $e_{f_{G_{3}}}(0)=\left(\begin{array}{l}t \\ 2\end{array}\right)+t^{2}+t^{2}+t+t+1, e_{f_{G_{3}}}(1)=\left(\begin{array}{l}t \\ 2\end{array}\right)+t$, $e_{f_{G_{3}}}(2)=\left(\begin{array}{c}t+1 \\ 2\end{array}\right)+t^{2}+t+t$. Also, the graphs $G_{l}$ and G $G_{3}$ gives $e_{f_{G_{1}}}(0)=t+1, \quad e_{f_{G_{1}}}(1)=t, e_{f_{G_{1}}}(2)=t \quad$ and $e_{f_{G_{2}}}(0)=t, e_{f_{G_{2}}}(1)=t, e_{f_{G_{2}}}(2)=t+1$. Totally, we have $e_{f}(0)=\frac{4+7 t+5 t^{2}}{2}, e_{f}(1)=\frac{5 t+t^{2}}{2}$,

$e_{f}(2)=\frac{2+9 t+3 t^{2}}{2}$

In this subcase, if we reduce the contribution $t^{2}+1$ from $e_{f}(0)$ and $t$ from $e_{f}(2)$, and add the contribution $t^{2}+t+1$ to $e_{f}(1)$, we get the condition (3). But, this removal affects the vertex labeling condition (2)..

Case (iii): $n \equiv 2(\bmod 3)$. Let $n=3 t+2$.

Now the graph $K_{1, n}+++$ has $6 t+5$ vertices and $\frac{14+27 t+9 t^{2}}{2}$ edges. We have 3 subcases.

(i) $v_{f}(0)=v_{f}(2)=2 t+2, \quad v_{f}(1)=2 t+1$.

(ii) $v_{f}(0)=v_{f}(1)=2 t+2, v_{f}(2)=2 t+1$.

(iii) $v_{f}(1)=v_{f}(2)=2 t+2, v_{f}(0)=2 t+1$ and

(a) $e_{f}(0)=\frac{5+9 t+3 t^{2}}{2}, e_{f}(1)=\frac{5+9 t+3 t^{2}}{2}$,

$e_{f}(2)=\frac{4+9 t+3 t^{2}}{2}$.

(b) $e_{f}(0)=\frac{5+9 t+3 t^{2}}{2}, e_{f}(2)=\frac{5+9 t+3 t^{2}}{2}$,

$e_{f}(1)=\frac{4+9 t+3 t^{2}}{2}$. 
(c) $e_{f}(1)=\frac{5+9 t+3 t^{2}}{2}, e_{f}(2)=\frac{5+9 t+3 t^{2}}{2}$,

$e_{f}(0)=\frac{4+9 t+3 t^{2}}{2}$.

Subcase $(\mathbf{i}): v_{f}(0)=v_{f}(2)=2 t+2, v_{f}(1)=2 t+1$.

Suppose the central vertex islabeled as 1.From Theorem 2.7 [7], this labeling does not affect the vertex labeling of $G_{1}$ and $G_{2}$, but from Theorem 2.1 [11], this labeling affects the vertex labeling of $G_{3}$.

Subcase (ii): $v_{f}(0)=v_{f}(1)=2 t+2, v_{f}(2)=2 t+1$.

Suppose the central vertex is labeled as 1 . From Theorem 2.7 [7] and from Theorem 2.1 [11], this labeling does not affect the vertex labeling of $G_{1}$ and $G_{3}$, but from Theorem 2.7 [7], this labeling affects the vertex labeling of $G_{2}$.

Subcase (iii): $v_{f}(1)=v_{f}(2)=2 t+2, v_{f}(0)=2 t+1$.

Suppose the central vertex islabeled as 1 . From Theorem 2.7 [7] and from Theorem 2.1 [11], this labeling does not affect the vertex labeling of $G_{l}$ and $G_{3}$, but from Theorem 2.7 [7], this labeling affects the vertex labeling of $G_{2}$.

Thus in all the above subcases, we see that $K_{1, n}{ }^{+++}$is not geometric mean cordial.

\section{REFERENCES}

[1] Albert William, Indra Rajasingh and S. Roy, "Mean cordial labeling of certain Graphs", " Open Journal of Computer Science and Mathematical Sciences,vol.4,(2013 ), 274 - 281.

[2] J. A. Bondy and U. S. R. Murthy, " Graph Theory with Applications, Newyork,North Holland 1976.

[3] I. Cahit, " Cordial Graphs", "A weaker version of graceful and harmonious Graphs" Ars. Combinatoria, vol. 23, No 3, 1987, pp, $201-207$.

[4] J. A. Gallian, “A Dynamic Survey of Graph Labeling”, vol 18, 2011, pp, 1-219.

[5] F. Harary, " Graph Theory “, Addison Wesley, New Delhi 1969.

[6] R. Ponraj, M. Sivakumar, " Mean Cordial Labeling of Graphs” , “ Open Journalof Discrete Mathematics”, vol 2, No 4, 2012, pp 145.

[7] K. Chitra Lakshmi , K. Nagarajan, " Geometric Mean Cordial Labeling of Graphs”, “International Journal of Mathematics and Soft Computing “, vol 7. No.1 (2017), pp $75-87$.

[8] K. Chitra Lakshmi , K. Nagarajan, "Geometric Mean Cordial Labeling Subdivison of Standard Graphs", International Journal of Pure and Applied Mathematics" Volume 112, No. 5, 2017, pp $103-$ 112.

[9] K. Chitra Lakshmi , K. Nagarajan, " Geometric Mean Cordial Labeling Corona of Standard Graphs", Proceedings in the International Multidisciplinary Research Foundation, India.", January 24 \& 25, 2017, pp $29-35$.

[10] K. Chitra Lakshmi , K. Nagarajan, " Geometric Mean Cordial Labeling Corona of Complete graph, Wheel and Complete Bipartite graph ", International Multidisciplinary Research Foundation, India ", December $14-16,2017$, pp $11-18$.

[11] K. Chitra Lakshmi , K. Nagarajan, " Geometric Mean Cordial Labeling of Transformation graphs of paths ", International Multidisciplinary Research Foundation, India”, Sep 06 -08, 2018, pp $223-235$.

[12] K. Chitra Lakshmi , K. Nagarajan, “ Geometric Mean Cordial Labeling of Transformation graphs of cycles ", International Multidisciplinary ResearchFoundation, India”, 30.10.2018 02.11.2018, pp $132-136$.

\section{AUTHORS PROFILE}

Dr. K. Nagarajan is working as a Professor of mathematics at Kalasalingamuniversity, Krishnankoil. He was working as Head and Associate Professorof Department of mathematics at Sri S.Ramasamy Naidu Memorial College, Sattur. He has guided 38 M.Phil. Scholars and 4 $\mathrm{Ph} . \mathrm{D}$. Scholars. He is currently guiding $5 \mathrm{Ph} . \mathrm{D}$. Scholars. Having published 11 research papers in national level journals and 40 research papers in international level journals.He has completed Minor Research Project sponsored by UGC.He has 9 years experience in research and his area of interests in research is graph labelling, domination of graphs, distances in graphs and energy of graphs.

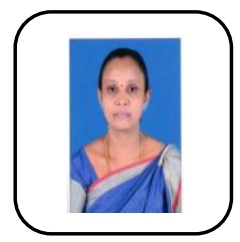

K. Chitra Lakshmi, \ Working as an Assistant Professor in Mathematics at V. V. Vanniaperumal College for Women, Virudhunagar. Working as Research Scholar for 4 years under the Guidance of Dr K. Nagarajan, Department of Mathematics Kalasalingam Academy of Research and Education Krishnankoil - 626126. 